\title{
Catalizadores $\mathrm{MnO}_{\mathrm{x}}-\mathrm{TiO}_{2}$ para la eliminación de compuestos orgánicos volátiles
}

\author{
J.M. GALLARDO AMORES ${ }^{1,3}$, V. SÁNCHEZ ESCRIBANO², G. BUSCA ${ }^{3}$ Y F. MILELLA ${ }^{3}$. \\ 1 Dep. Química Inorgánica. Fa Ciencias Químicas. Universidad Complutense. 28040 Ciudad Universitaria (Madrid). \\ 2 Dep. Química Inorgánica. Fª Ciencias Químicas. Universidad de Salamanca. Pa Merced, s/n, 37008 Salamanca \\ 3 Istituto di Chimica. Facoltà di Ingegneria. Università di Genova. Ple Kennedy. Fiera di Mare. 16129 Génova (Italia).
}

\begin{abstract}
Se han sintetizado muestras de $\mathrm{Mn}$-Ti de fórmula general $\mathrm{Ti}_{\mathrm{x}} \mathrm{Mn}_{1-\mathrm{x}} \mathrm{O}_{\mathrm{y}}(\mathrm{x}=0,0.1,0.2,0.5,0.8,0.9,1)$ mediante un método de coprecipitación. Las fases cristalinas mayoritarias identificadas fueron $\mathrm{Mn}_{3} \mathrm{O}_{4}$ (hausmanita) y $\mathrm{TiO}_{2}$ (anatasa), formándose también disoluciones sólidas a bajos porcentajes de uno de los cationes. Los materiales son estables térmicamente hasta los $873 \mathrm{~K}$, tranformándose en las fases termodinámicamente estables, $\mathrm{TiO}_{2}$ (rutilo) y $\alpha-\mathrm{Mn}_{2} \mathrm{O}_{3}$ a mayores temperaturas. Desde el punto de vista catalítico, son activos en la oxidación de propano y propeno, aunque el aumento del contenido de Ti parece desactivar el óxido de Mn en la oxidación de propileno. Contrariamente, en la oxidación de propano la relación Mn/Ti=1 presenta un máximo en la conversión de propano.
\end{abstract}

Palabras clave: VOC, disolución sólida, anatasa, oxidación catalítica, hausmanita.

$\mathrm{MnOx}-\mathrm{TiO} 2$ catalyzers for valatil organic compounds elimination.

Mn-Ti mixed oxides with stoichiometry $\mathrm{Ti}_{\mathrm{x}} \mathrm{Mn}_{1-\mathrm{x}} \mathrm{O}_{\mathrm{y}}$ (with $\mathrm{x}=0,0.1,0.2,0.5,0.8,0.9,1$ ) have been prepared by a coprecipitation method. $\mathrm{Mn}_{3} \mathrm{O}_{4}$ (hausmannite) and $\mathrm{TiO}_{2}$ (anatase) were found to be the main crystalline phases, while solid solutions appeared to be formed at the extreme values of $x$. Thermal stability of these materials reaches $873 \mathrm{~K}$, transforming into thermodynamically stable phases, $\mathrm{TiO}_{2}$ (rutile) y $\alpha-\mathrm{Mn}_{2} \mathrm{O}_{3}$, at higher temperatures. From catalytic point of view, they are active in propane and propene oxidation, though the increase of $\mathrm{Ti}$ content seems to deactivate $\mathrm{Mn}$ oxide for propene oxidation. Conversely, the sample with the $\mathrm{Mn} / \mathrm{Ti}=1$ ratio presents a maximum for propane oxidation.

Key words: VOC catalyzers, solid solution, anathase, catalytic oxidation, hausmanite.

\section{INTRODUCCIÓN}

Los óxidos de manganeso, tanto puros como en forma de óxidos mixtos de formulación más compleja, constituyen un tipo de catalizadores ampliamente utilizados por sus notables propiedades oxidativas. Así, estos materiales en sus diferentes estructuras: manganitos de tipo perovskita $\left({ }^{1}\right), \beta$-aluminas conteniendo $\mathrm{Mn}\left({ }^{2}\right)$, espinelas con $\mathrm{Mn}\left({ }^{3}\right)$, varios óxidos de $\mathrm{Mn}$ soportados sobre alumina en forma de polvo $\left({ }^{4}\right)$ y sobre monolitos de cordierita $\left(^{5}\right)$ y también los óxidos puros $\mathrm{MnO}_{2}(6,7,8)$, $\mathrm{Mn}_{2} \mathrm{O}_{3}\left({ }^{9},{ }^{10}\right)$ and $\mathrm{Mn}_{3} \mathrm{O}_{4}\left({ }^{11}\right)$, se han propuesto como catalizadores en la eliminación de compuestos orgánicos volátiles (VOC) y en la combustión total de metano. Respecto a otros catalizadores, presentan la ventaja de ser menos contaminantes con el medio ambiente y más selectivos respecto a los tradicionales formados por metales nobles soportados sobre un óxido cerámico (12). El principal inconveniente es su menor actividad.

Desde el punto de vista medioambiental, los óxidos mixtos de Mn-Ti y Mn-Al, preparados por varios métodos, también se han propuesto como catalizadores en la oxidación selectiva de $\mathrm{NH}_{3}$ a $\mathrm{N}_{2}$ (13) y la reducción catalítica selectiva (SCR) de $\mathrm{NO}$ a $\mathrm{N}_{2}$ mediante amoníaco (14).

Por tanto, debido a su interés en el campo de la catálisis, en los últimos años se han realizado notables esfuerzos orientados a mejorar sus propiedades. Así mismo, teniendo en cuenta el conocido efecto de activación que produce el óxido de titanio sobre la fase activa que soporta en catalizadores comerciales SCR formados por V-W-Ti (15), parece razonable incidir en la síntesis de sistemas mixtos Mn-Ti para observar las posibles sinergias entre ambos metales.

En este trabajo se presentan los resultados obtenidos en la caracterización de óxidos mixtos complejos de $\mathrm{Mn}$-Ti sintetizados mediante un método de coprecipitación, así como un estudio de la actividad catalítica de estos óxidos en la transformación de hidrocarburos orgánicos volátiles (VOC).

\section{PARTE EXPERIMENTAL}

\subsection{Preparación de las muestras}

Los óxidos mixtos de $\mathrm{Mn}$-Ti con estequiometría $\mathrm{Ti}_{\mathrm{x}} \mathrm{Mn}_{1-\mathrm{x}} \mathrm{O}_{\mathrm{y}}$ $(\mathrm{x}=0,0.1,0.2,0.5,0.8,0.9,1)$ se prepararon mezclando una solución acuosa de $\mathrm{Mn}\left(\mathrm{CH}_{3} \mathrm{COO}\right)_{3} .2 \mathrm{H}_{2} \mathrm{O}$ (Acros, $98 \%$ ) con las cantidades requeridas $\mathrm{y}$ volúmenes precisos de Ti $\left[\mathrm{OCH}\left(\mathrm{CH}_{3}\right)_{2}\right]_{4}$ (Aldrich, 97\%) en agitación continua a $333 \mathrm{~K}$ durante $24 \mathrm{~h}$. El gel se secó $24 \mathrm{~h}$ a $393 \mathrm{~K}$ y finalmente se calcinó en aire $4 \mathrm{~h}$ a $773 \mathrm{~K}$.

\subsection{Técnicas experimentales}

Los difractogramas de rayos $\mathrm{X}$ se registraron en un difractómetro Philips PW 1710 (radiación $\mathrm{Cu} \mathrm{K \alpha}$, filtro Ni; 45kV, $35 \mathrm{~mA}$ ). Los parámetros de celda se calcularon mediante un 
preciso software de mínimos cuadrados.

Los espectros FT-IR se registraron con un espectrómetro Nicolet Magna 750 con transformada de Fourier en la región $4000-50 \mathrm{~cm}^{-1}$

Los espectros FT-Raman se registraron con un espectrómetro Brucker RFS100, con un láser de Nd-YAG (1064 nm), con una potencia de $30 \mathrm{~mW}, 2000$ scans y una resolución de $4 \mathrm{~cm}^{-1}$.

Las superficies específicas se midieron con un aparato volumétrico convencional utilizando nitrógeno como adsorbato a $77 \mathrm{~K}$.

Los análisis térmicos (DTA-TG) se realizaron, en atmósfera de aire, con una termobalanza Setaram TGA 92-12, desde temperatura ambiente hasta $1273 \mathrm{~K}$ a la velocidad de calentamiento de $10 \mathrm{~K} / \mathrm{min}$.

\subsection{Ensayos catalíticos}

Los ensayos catalíticos de las reacciones de oxidación de propano y propeno se realizaron en un reactor tubular con un flujo total de $330 \mathrm{ml} / \mathrm{min}$. Las condiciones de preparación del catalizador y la metodología experimental se han descrito previamente (11).

\section{RESULTADOS Y DISCUSIÓN}

\subsection{Caracterización de las muestras}

En la Tabla I se resumen los principales datos morfológicos y estructurales de las muestras. En los difractogramas de los óxidos mixtos de Mn-Ti (Fig.1, c-e)) se identifican las fases $\mathrm{Mn}_{3} \mathrm{O}_{4}$ (hausmanita, espinela con distribución aleatoria de los cationes) y $\mathrm{TiO}_{2}$ (anatasa) bien cristalizadas. Las intensidades de ambas fases guardan una relación directa con el contenido del catión correspondiente. Para las muestras con bajo contenido en uno de los dos cationes, $\mathrm{Ti}_{0.1} \mathrm{Mn}_{0.9}$ y $\mathrm{Ti}_{0.9} \mathrm{Mn}_{0.1}$, sólo se observa la fase hausmanita o la fase anatasa (Fig. 1, b,f), respectivamente; indicando, claramente, que el catión que se encuentra en menor proporción difunde hacia el interior de la estructura de la fase mayoritaria formando una disolución

TABLA I. DATOS MORFOLÓGICO Y ESTRUCTURALES DE LAS MUESTRAS $\mathrm{Ti}_{\chi} \mathrm{Mn}_{1-\mathrm{x}} \mathrm{O}_{Y}$ A $773 \mathrm{~K}$

\begin{tabular}{|c|c|c|c|c|c|}
\hline \multirow[t]{2}{*}{ Muestra } & \multirow[t]{2}{*}{ Fases } & \multicolumn{2}{|c|}{ 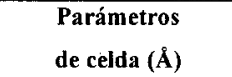 } & \multirow[t]{2}{*}{ Volume $\left(\AA^{3}\right)$} & \multirow[t]{2}{*}{ BET $\left(\mathrm{m}^{2} / \mathrm{g}\right)$} \\
\hline & & a & c & & \\
\hline $\mathrm{Ti}$ & $\begin{array}{c}\text { Anatasa } \\
\text { (Brookita) }\end{array}$ & $3.777(2)$ & $9.460(6)$ & 135.0 & 94 \\
\hline $\mathrm{Ti}_{0.9} \mathrm{Mn}_{0.1} \mathrm{O}_{y}$ & Anatasa & $3.790(1)$ & $9.446(5)$ & 135.6 & 83 \\
\hline $\mathrm{Ti}_{0.8} \mathrm{Mn}_{0.2} \mathrm{O}_{\mathrm{y}}$ & $\begin{array}{c}\text { Anatasa } \\
\text { (Hausmanita) }\end{array}$ & $3.790(1)$ & $9.493(4)$ & 136.4 & 53 \\
\hline $\mathrm{Ti}_{0.5} \mathrm{Mn}_{0.5} \mathrm{O}_{\mathrm{y}}$ & $\begin{array}{c}\text { Anatasa } \\
\text { Hausmanita } \\
\text { (Rutilo) }\end{array}$ & $\begin{array}{l}3.809(6) \\
5.763(1)\end{array}$ & $\begin{array}{l}9.434(27) \\
9.429(5)\end{array}$ & $\begin{array}{l}136.9 \\
313.0\end{array}$ & 42 \\
\hline $\mathrm{Ti}_{0.2} \mathrm{Mn}_{0.8} \mathrm{O}_{\mathrm{y}}$ & $\begin{array}{l}\text { Hausmanita } \\
\text { (Anatasa) }\end{array}$ & $5.761(0)$ & $9.427(3)$ & 313.0 & 25 \\
\hline $\mathrm{Ti}_{0.1} \mathrm{Mn}_{0.9} \mathrm{O}_{\mathrm{y}}$ & Hausmanita & $5.768(0)$ & $9.446(1)$ & 314.3 & 18 \\
\hline $\mathrm{Mn}$ & Hausmanita & $5.759(1)$ & $9.443(5)$ & 314.3 & 11 \\
\hline
\end{tabular}

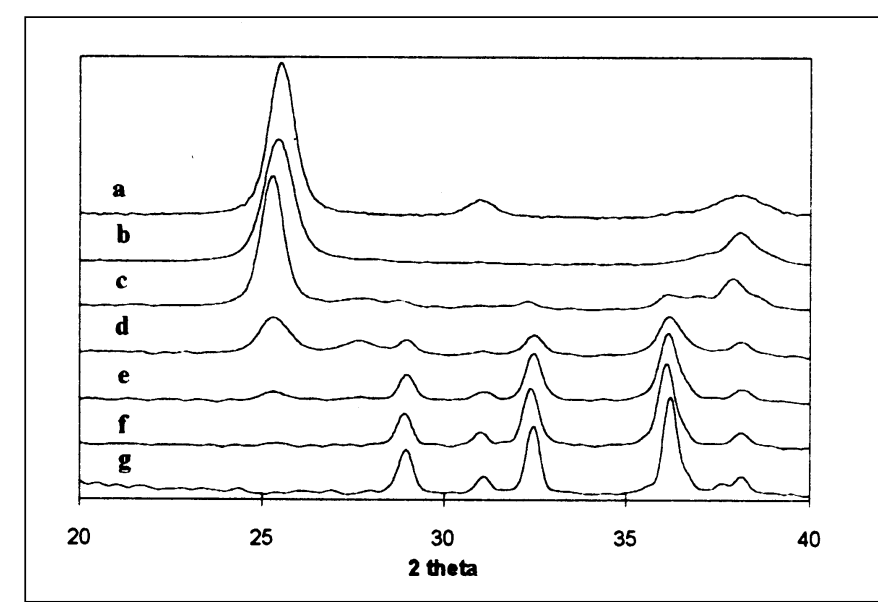

Fig. 1. Difractogramas de RX de las muestras calcinadas a $773 \mathrm{~K}$. a) $\mathrm{TiO}_{2}$, b) $\mathrm{Ti}_{0.9} \mathrm{Mn}_{0.1} \mathrm{O}_{\mathrm{y}^{\prime}}$ c) $\mathrm{Ti}_{0.8} \mathrm{Mn}_{0.2} \mathrm{O}_{\mathrm{y}^{\prime}}$ d) $\mathrm{Ti}_{0.5} \mathrm{Mn}_{0.5} \mathrm{O}_{\mathrm{y}^{\prime}}$ e) $\mathrm{Ti}_{0.2} \mathrm{Mn}_{0.8} \mathrm{O}_{\mathrm{y}^{\prime}}$ f) $\mathrm{Ti}_{0.1} \mathrm{Mn}_{0.9} \mathrm{O}_{\mathrm{y}^{\prime}}$ g) $\mathrm{Mn}_{3} \mathrm{O}_{4}$.

sólida. Los parámetros de celda y el volumen calculados para la fase anatasa muestran un crecimiento continuo del volumen, mientras que para la fase hausmanita esta tendencia no está tan definida, permaneciendo el volumen prácticamente constante. Este comportamiento es debido, posiblemente, mediante la diferencia entre los radios iónicos de los cationes implicados $\mathrm{Mn}^{2+}(80 \mathrm{pm}), \mathrm{Mn}^{3+}(66 \mathrm{pm})$ y $\mathrm{Ti}^{4+}$ (74 pm). Así, al difundir Mn en la estructura de la anatasa, ésta será deformada mucho más que en el caso contrario, puesto que además se suma el efecto aleatorio de la distribución de los cationes $\mathrm{Mn}^{2+}$ y $\mathrm{Mn}^{3+}$. Este último amortigua, en parte, la posible deformación debida al $\mathrm{Mn}^{2+}$. Respecto a las áreas tienden a disminuir con el aumento del contenido en $\mathrm{Mn}$, debido probablemente a

TABLA II. DATOS TERMOGRAVIMÉTRICOS DE LAS MUESTRAS $\mathrm{Ti}_{\mathrm{x}} \mathrm{Mn}_{1-\mathrm{x}} \mathrm{O}_{\mathrm{y}}$

\begin{tabular}{|c|c|c|c|}
\hline Muestras & Temperatura (K) & Pérdida de peso (\%) & Ganancia de peso $(\%)$ \\
\hline $\mathrm{TiO}_{2}$ & $\begin{array}{c}873-973 \\
1013\end{array}$ & - & - \\
\hline $\mathrm{Ti}_{0.9} \mathrm{Mn}_{0.1} \mathrm{O}_{\mathrm{y}}$ & $\begin{array}{l}963 \\
1203\end{array}$ & $\begin{array}{c}- \\
0.65\end{array}$ & - \\
\hline $\mathrm{Ti}_{0.8} \mathrm{Mn}_{0.2} \mathrm{O}_{\mathrm{y}}$ & $\begin{array}{l}963 \\
1203\end{array}$ & $\begin{array}{c}- \\
0.92\end{array}$ & $\begin{array}{l}- \\
-\end{array}$ \\
\hline $\mathrm{Ti}_{0.5} \mathrm{Mn}_{0.5} \mathrm{O}_{\mathrm{y}}$ & $\begin{array}{c}873-963 \\
963 \\
1203\end{array}$ & $\begin{array}{c}0.80 \\
- \\
2.61\end{array}$ & $\begin{array}{c}- \\
0.45 \\
-\end{array}$ \\
\hline $\mathrm{Ti}_{0.2} \mathrm{Mn}_{0.8} \mathrm{O}_{\mathrm{y}}$ & $\begin{array}{c}863-1023 \\
1203\end{array}$ & $\begin{array}{c}- \\
0.45\end{array}$ & $\begin{array}{c}0.90 \\
-\end{array}$ \\
\hline $\mathrm{Ti}_{0.1} \mathrm{Mn}_{0.9} \mathrm{O}_{\mathrm{y}}$ & $\begin{array}{c}863-1057 \\
1203\end{array}$ & $\begin{array}{c}- \\
0.30\end{array}$ & $\begin{array}{c}1.70 \\
-\end{array}$ \\
\hline $\mathrm{Mn}_{3} \mathrm{O}_{4}$ & $\begin{array}{c}863 \\
1057\end{array}$ & $\begin{array}{c}- \\
1.40\end{array}$ & $\begin{array}{c}2.05 \\
-\end{array}$ \\
\hline
\end{tabular}

Boletín de la Sociedad Española de Cerámica y Vidrio. Vol. 38 Núm. 3 Mayo-Junio 1999 
la ocupación de la superficie y parte de los huecos existentes en la estructura por el Mn.

Los datos de los termogramas se muestran en la Tabla II y las curvas DTA en el intervalo 673-1273 K se comparan en la Fig. 2. En la muestra pura de $\mathrm{TiO}_{2}$ se observa un pico exotérmico neto que se relaciona con la transición de fase anatasa-rutilo del óxido de titanio. Este máximo se ve influenciado por la presencia de Mn en la estructura, desplazándose hacia temperaturas más bajas, llegando a situarse a $963 \mathrm{~K}$ para la muestra $\mathrm{Ti}_{0.8} \mathrm{Mn}_{0.2} \mathrm{O}_{\mathrm{y}}$ (Fig. 2,c). Para el resto de las muestras, este máximo se solapa con otras características exotérmicas haciendo la interpretación más compleja. A partir de la muestra $\mathrm{Ti}_{0.5} \mathrm{Mn}_{0.5} \mathrm{O}_{\mathrm{y}}$ (Fig. 2,d), se observa una zona de pérdida de peso hasta $963 \mathrm{~K}$, seguida de una posterior ganancia para volver a perder peso en torno a $1203 \mathrm{~K}$. Estos datos se relacionan con las transformaciones que sufren los óxidos de $\mathrm{Mn}$ : i) primero el Mn es segregado durante la transición de fase anatasa-rutilo, ii) posteriormente se oxida obteniéndose $\alpha-\mathrm{Mn}_{2} \mathrm{O}_{3}$ (fase termodinámicamente estable) y iii) finalmente, a altas temperaturas reacciona con el $\mathrm{TiO}_{2}$ (rutilo) para dar $\mathrm{MnTiO}_{3}$ (pirofanita). Los cálculos teóricos de variación de peso asociados a estas reacciones coinciden, en buena aproximación, con los experimentales. La formación final de pirofanita lleva asociado un pico endotérmico que aumenta con el contenido de Mn (Fig. 2,d-f). Por tanto podemos concluir que los catalizadores son estables hasta $873 \mathrm{~K}$, sufriendo posteriormente transformaciones estructurales. Además, el Mn parece favorecer la transición de fase anatasa-rutilo, al igual que ocurre con otros metales de la primera serie de transición (16).

\subsection{Caracterización espectroscópica de los materiales catalíticos}

Los espectros esqueletales en la zona de $1200-50 \mathrm{~cm}^{-1}$ muestran las absorciones típicas de la fase espinela hausmanita para el óxido puro de Mn con bandas principales a 611, 421, 245, 165 y $124 \mathrm{~cm}^{-1}$. Los números de onda asignados corresponden aproximadamente con los aportados por Lutz et al. $\left({ }^{17}\right)$. Estas bandas van perdiendo intensidad y haciéndose más anchas al disminuir el contenido en $\mathrm{Mn}$, hasta hacerse indistinguibles en la muestra $\mathrm{Ti}_{0.5} \mathrm{Mn}_{0.5} \mathrm{O}_{\mathrm{y}}$. Paralelamente, las características del $\mathrm{TiO}_{2}$ (anatasa) con bandas anchas en la zona $800-400 \mathrm{~cm}^{-1}\left({ }^{18}\right)$ van apareciendo a partir de esta muestra hasta hacerse predominantes. Al igual que sucedía en XRD en la muestra de $\mathrm{TiO}_{2}$, se puede observar una situación más compleja en la zona 600$400 \mathrm{~cm}^{-1}$ debida a la presencia de brookita.

De forma análoga, los espectros Raman de los óxidos (Fig. 3) muestran los picos asociados a los modos de vibración del $\mathrm{TiO}_{2}$ (anatasa) muy intensos y nítidos a 638, 512, 395, 322, 236 y $142 \mathrm{~cm}^{-1}$, junto a otras más débiles, debidas a impurezas de brookita, a 450 y $359 \mathrm{~cm}^{-1}$ (18) para la muestra sin $\mathrm{Mn}$ y las posteriores hasta $\mathrm{Ti}_{0.2} \mathrm{Mn}_{0.8} \mathrm{O}_{\mathrm{y}}$ (Fig. 3, a-c). Así mismo, se produce un decrecimiento de la intensidad debida al recubrimiento de la superficie por parte del óxido de Mn, ando lugar a un descenso progresivo del área superficial como se comentó anteriormente. En la Fig. 3,d comienza a observarse el pico característico de la fase hausmanita situado a $655 \mathrm{~cm}^{-1}$, el cual aumenta progresivamente con el contenido de Mn (Fig. 3,e-g).

Los resultados obtenidos mediante las técnicas espectroscópicas confirman los datos XRD mostrados en la Tabla I.

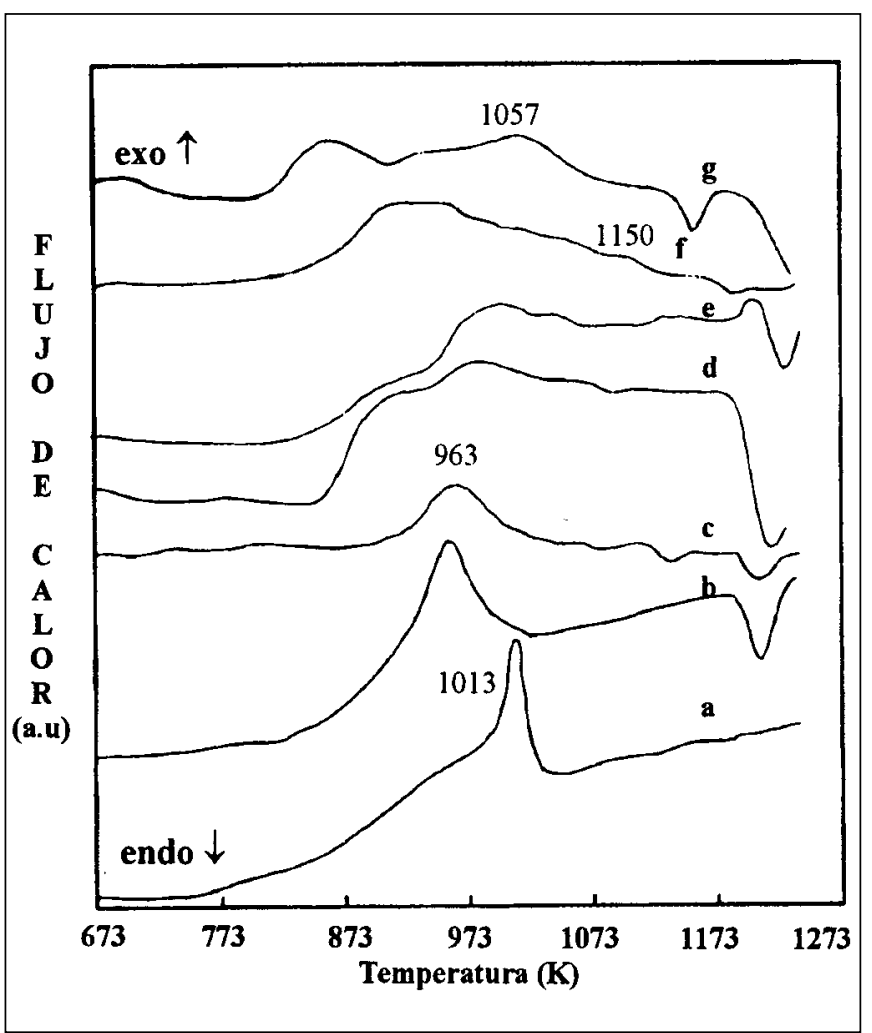

Fig. 2. Curvas DTA de las muestras. a) $\mathrm{TiO}_{2}$, b) $\mathrm{Ti}_{0.9} \mathrm{Mn}_{0.1} \mathrm{O}_{\mathrm{y}^{\prime}}$ c) $\mathrm{Ti}_{0.8} \mathrm{Mn}_{0.2} \mathrm{O}_{\mathrm{y}^{\prime}}$ d) $\mathrm{Ti}_{0.5} \mathrm{Mn}_{0.5} \mathrm{O}_{\mathrm{y}^{\prime}}$ e) $\mathrm{Ti}_{0.2} \mathrm{Mn}_{0.8} \mathrm{O}_{\mathrm{y}^{\prime}}$ f) $\mathrm{Ti}_{0.1} \mathrm{Mn}_{0.9} \mathrm{O}_{\mathrm{y}^{\prime}}$ g) $\mathrm{Mn}_{3} \mathrm{O}_{4}$.

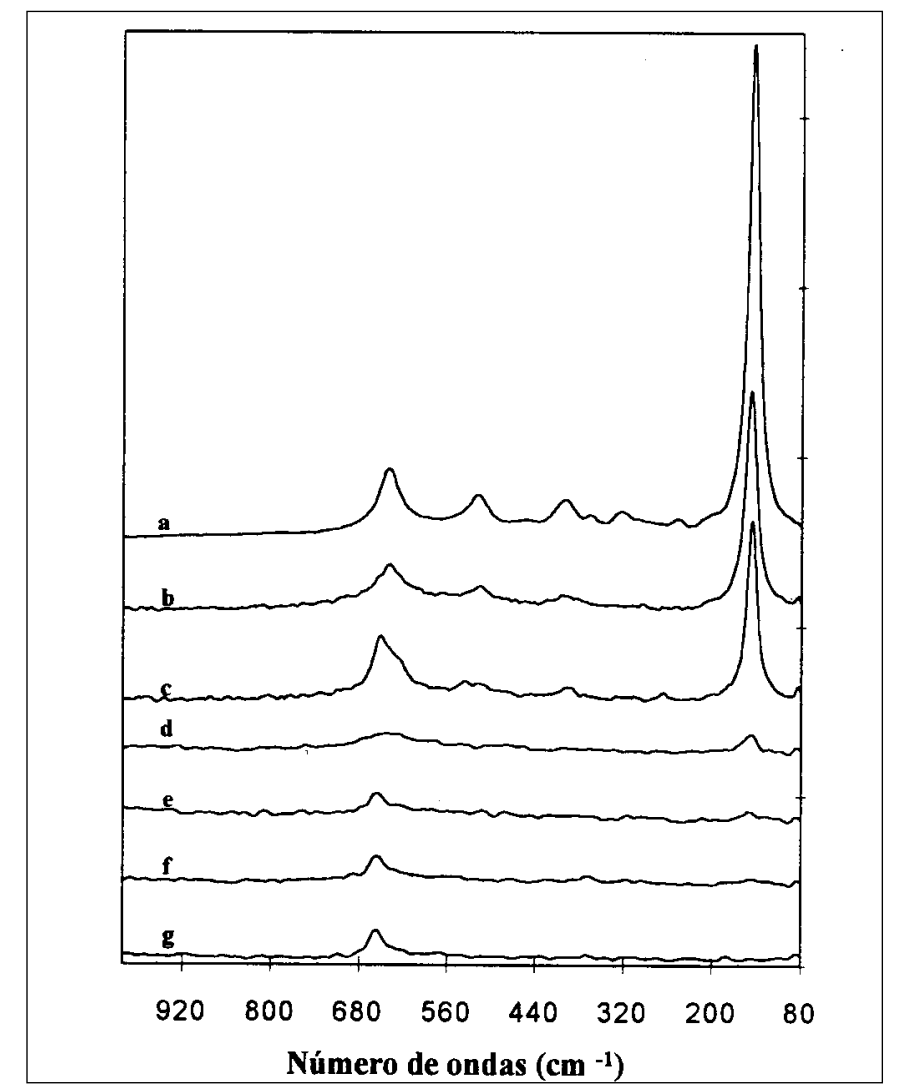

Fig. 3. Espectros FT-Raman de las muestras calcinadas a $773 \mathrm{~K}$. a) $\mathrm{TiO}_{2}$, b) $\mathrm{Ti}_{0.9} \mathrm{Mn}_{0.1} \mathrm{O}_{\mathrm{y}^{\prime}}$ c) $\mathrm{Ti}_{0.8} \mathrm{Mn}_{0.2} \mathrm{O}_{\mathrm{y}^{\prime}}$ d) $\mathrm{Ti}_{0.5} \mathrm{Mn}_{0.5} \mathrm{O}_{\mathrm{y}^{\prime}}$ e) $\mathrm{Ti}_{0.2} \mathrm{Mn}_{0.8} \mathrm{O}_{\mathrm{y}^{\prime}}$ f) $\mathrm{Ti}_{0.1} \mathrm{Mn}_{0.9} \mathrm{O}_{\mathrm{y}^{\prime}}$ g) $\mathrm{Mn}_{3} \mathrm{O}_{4}$. 


\subsection{Oxidación de propano}

Todas las muestras presentan actividad en la reacción de oxidación de propano variando el intervalo de temperaturas que comprende el comienzo de la reacción y la combustión total a $\mathrm{CO}_{2}$. En la Fig. 4 se observa la evolución de la reacción para la muestra $\mathrm{Ti}_{0.1} \mathrm{Mn}_{0.9} \mathrm{O}_{\mathrm{y}}$ mostrándose los principales productos detectados mediante cromatografía de gases. La reacción comienza en torno a $475 \mathrm{~K}$ obteniéndose propileno principalmente hasta $525 \mathrm{~K}$. A partir de esta temperatura, se comienza a producir $\mathrm{CO}_{2}, \mathrm{CO}(<0.06 \%)$ y etileno $(<12 \%)$ como consecuencia del cracking de la molécula. Claramente, se comienza a perder selectividad y se favorece la combustión total. No obstante, la selectividad hacia propileno se mantiene en torno al 35\% para conversiones de propano del $15 \%$. Tanto el intervalo de actividad como el de selectividades son notablemente sensibles al contenido de Mn. Este hecho puede observarse en la Fig. 5 donde se representan las conversiones de propano en función de la temperatura para todas las muestras obtenidas. El óxido más activo es el $\mathrm{Mn}_{2} \mathrm{O}_{3}$, el cual alcanza la combustión total en torno a $550 \mathrm{~K}$, mientras el resto, incluido el $\mathrm{Mn}_{3} \mathrm{O}_{4}$, van extendiendo el intervalo de temperaturas hasta superar los $750 \mathrm{~K}$. La influencia del Ti se puede observar fácilmente en la Fig. 6, donde se representan las selectividades y conversiones en función de la composición de la muestra a la temperatura de $600 \mathrm{~K}$. Para esta reacción los óxidos mixtos de $\mathrm{Mn}$-Ti son menos activos que $\mathrm{Mn}_{2} \mathrm{O}_{3}$ y $\mathrm{Mn}_{3} \mathrm{O}_{4}$ y entre ellos parece que la estructura influye notablemente, encontrándose un máximo en la conversión de propano para la muestra $\mathrm{Ti}_{0.5} \mathrm{Mn}_{0.5} \mathrm{O}_{\mathrm{y}}$. En cuanto a la selectividad hacia propileno, esta se hace máxima para la muestra, situada en el campo de las disoluciones sólidas, $\mathrm{Ti}_{0.2} \mathrm{Mn}_{0.8} \mathrm{O}_{\mathrm{y}}$. Por tanto, se deduce que la interacción entre ambos cationes favorece la selectividad en esta reacción.

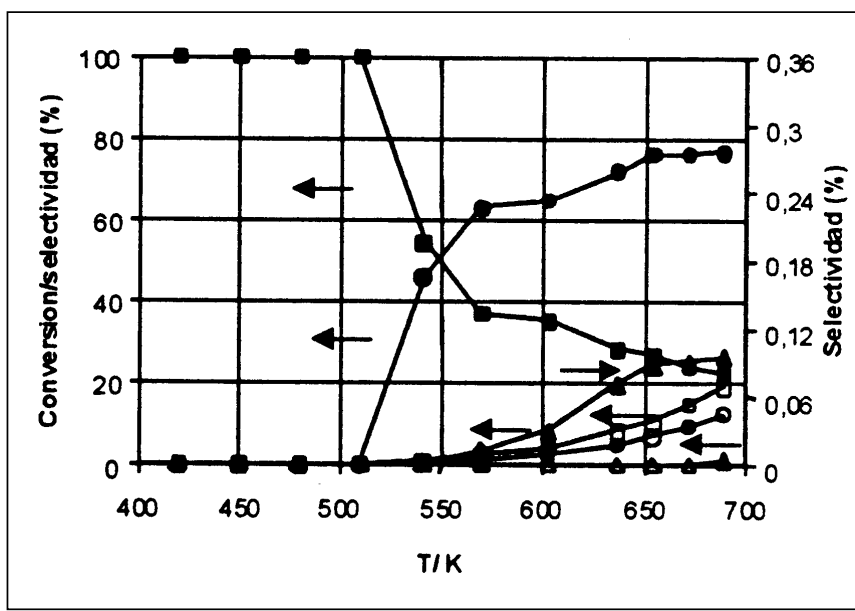

Fig. 4. Conversión de propano y selectividades en función de la temperatura para la muestra Ti0.1Mn0.9Oy en la reacción de oxidación de propano. $\mathrm{O}_{2}(\mathrm{C})(\mathbf{O}), \mathrm{CO}_{2}(\mathrm{~S})(\bullet), \mathrm{C}_{3} \mathrm{H}_{8}(\mathrm{C})(\square), \mathrm{C}_{3} \mathrm{H}_{6}(\mathrm{~S})(\bullet), \mathrm{CO}$ (S) $(\Delta), \mathrm{C}_{2} \mathrm{H}_{4}(\mathrm{~S})(\mathbf{\Delta})$.

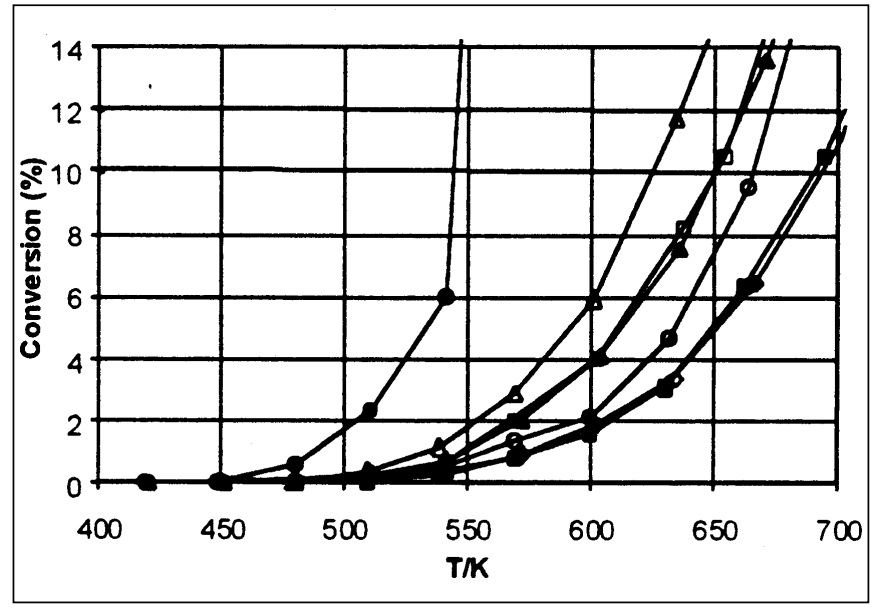

Fig. 5. Conversión de propano en función de la temperatura para las muestras. $\mathrm{Mn}_{3} \mathrm{O}_{4}(\mathbf{O}), \mathrm{Mn}_{3} \mathrm{O}_{4}(\bullet), \mathrm{Ti}_{0.1} \mathrm{Mn}_{0.9} \mathrm{O}_{\mathrm{y}}(\boldsymbol{\square}), \mathrm{Ti}_{0.2} \mathrm{Mn}_{0.8} \mathrm{O}_{\mathrm{y}}(\mathbf{\square})$, $\mathrm{Ti}_{0.5} \mathrm{Mn}_{0.5} \mathrm{O}_{\mathrm{y}}(\Delta), \mathrm{Ti}_{0.8} \mathrm{Mn}_{0.2} \mathrm{O}_{\mathrm{y}}(\boldsymbol{\Delta}), \mathrm{Ti}_{0.9} \mathrm{Mn}_{0.1} \mathrm{O}_{\mathrm{y}}(\diamond)$.

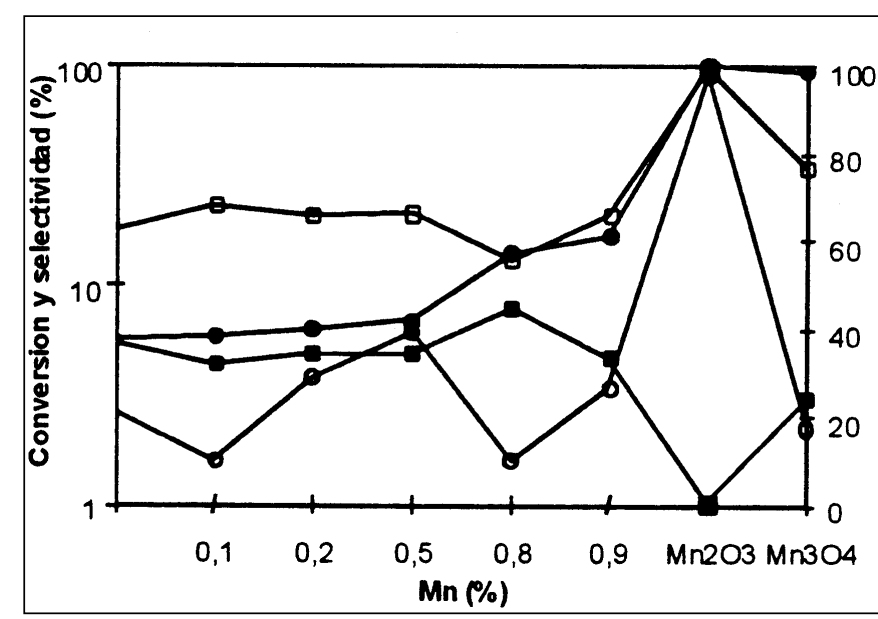

Fig. 6. Conversiones y selectividades en función de la composición de $\mathrm{Mn}$ a la temperatura de $600 \mathrm{~K} . \mathrm{C}_{3} \mathrm{H}_{8}(\mathrm{C})(\mathbf{0}), \mathrm{C}_{3} \mathrm{H}_{6}(\mathrm{C})(\bullet), \mathrm{CO}_{2}(\mathrm{~S})$ (ㅁ), $\mathrm{C}_{3} \mathrm{H}_{6}(\mathrm{~S})(\mathbf{\square})$

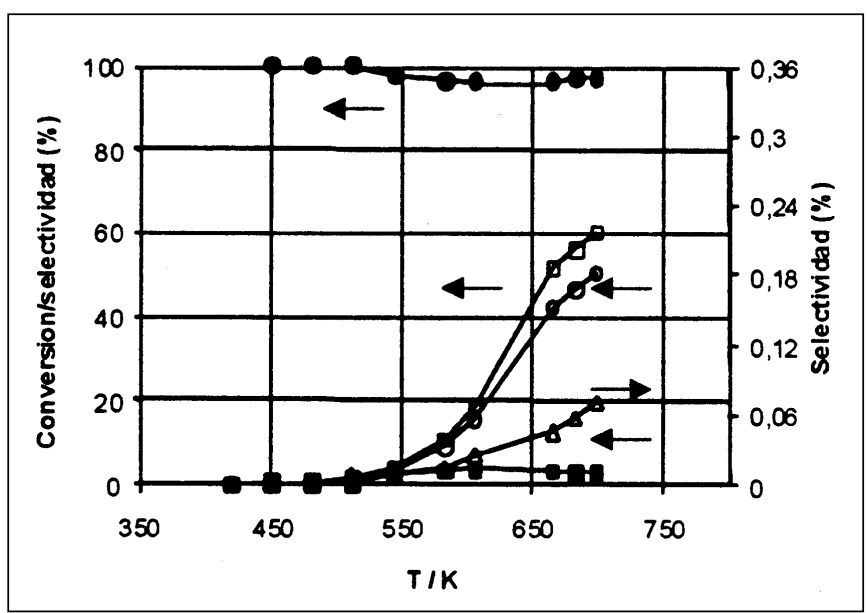

Fig. 7. Conversión de propileno y selectividades en función de la temperatura para la muestra $\mathrm{Ti}_{0.1} \mathrm{Mn}_{0.9} \mathrm{O}_{\mathrm{y}}$ en la reacción de oxidación de propileno. $\mathrm{O}_{2}(\mathrm{C})(\mathbf{O}), \mathrm{CO}_{2}(\mathrm{~S})(\bullet), \mathrm{C}_{3} \mathrm{H}_{6}(\mathrm{C})(\square), \mathrm{CO}(\mathrm{S})(\mathbf{\bullet}), \mathrm{C}_{2} \mathrm{H}_{4}(\mathrm{~S})(\Delta)$. 


\subsection{Oxidación de propano}

En la Fig. 7 se muestra la evolución de la reacción de oxidación de propeno para la muestra $\mathrm{Ti}_{0.1} \mathrm{Mn}_{0.9} \mathrm{O}_{\mathrm{y}}$. En este caso, la actividad aumenta considerablemente debido a la mayor labilidad de este compuesto. De este modo, el intervalo de temperatura de combustión total se reduce notablemente y se sitúa entre 435 y $625 \mathrm{~K}$. El producto principal es $\mathrm{CO}_{2}$, reduciéndose significativamente las concentraciones de $\mathrm{CO}$ y $\mathrm{C}_{2} \mathrm{H}_{4}(<0.06)$. La comparación de las conversiones de propeno frente a la temperatura (Fig. 8) muestra una variación continua con el contenido de Ti, el cual tiende a disminuir la actividad de las muestras. Por otra parte, los óxidos puros de manganeso no presentan diferencias significativas. Este mismo comportamiento se puede observar en la Fig. 6, donde la conversión de propileno a temperatura constante $(600 \mathrm{~K})$ aumenta con el contenido de Mn.

A partir de estos datos se puede deducir, que el óxido de titanio no potencia el efecto catalítico del óxido de manganeso en reacciones de oxidación de compuestos lábiles, como el propeno, pero en cambio, en pequeñas cantidades, puede mejorar la selectividad de propano hacia propileno en el intervalo de bajas conversiones, como se deduce del análisis de la Fig. 6.

\section{CONCLUSIONES}

Las conclusiones principales de este trabajo pueden resumirse de la siguiente forma:

1. Se ha comprobado que el método de coprecipitación controlada permite obtener óxidos mixtos de Mn-Ti con disoluciones sólidas para composiciones inferiores al 10\% de uno de los dos cationes.

2. La adición de Mn favorece la transición de fase anatasarutilo del óxido de titanio.

3. Los catalizadores son estables hasta la temperatura de $873 \mathrm{~K}$.

4. Las superficies específicas de los óxidos mixtos de Mn-Ti tienden a disminuir debido al progresivo recubrimiento de la superficie por parte del Mn, que produce, a su vez, una acusada disminución del efecto Raman como se observó en los espectros.

5. Los catalizadores presentan actividad en la combustión

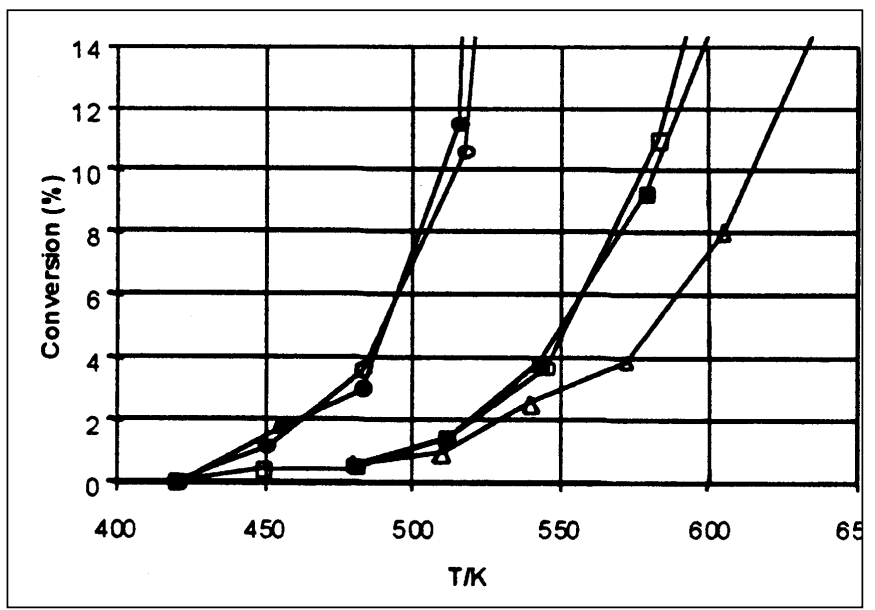

Fig. 8. Conversión de propileno en función de la temperatura para las muestras. $\mathrm{Mn}_{3} \mathrm{O}_{4}(\mathbf{O}), \mathrm{Mn}_{3} \mathrm{O}_{4}(\bullet), \mathrm{Ti}_{0.1} \mathrm{Mn}_{0.9} \mathrm{O}_{\mathrm{y}}(\square), \mathrm{Ti}_{0.2} \mathrm{Mn}_{0.8} \mathrm{O}_{\mathrm{y}}$ (匹), $\mathrm{Ti}_{0.5} \mathrm{Mn}_{0.5} \mathrm{O}_{\mathrm{y}}(\Delta)$. total de hidrocarburos ligeros y cierta selectividad desde propano hacia propileno, en función de sus composiciones.

6. La progresiva adición de Ti provoca la desactivación de los catalizadores en la reacción de oxidación de propeno.

7. Para bajos contenidos de Ti la selectividad hacia propileno desde propano aumenta respecto a los óxidos de Mn puros, de los cuales el más activo es el $\alpha-\mathrm{Mn}_{2} \mathrm{O}_{3}$.

La activación catalítica que presentan ciertos óxidos de transición $\left(\mathrm{V}_{2} \mathrm{O}_{5}, \mathrm{MnO}_{3}\right.$ y $\left.\mathrm{WO}_{3}\right)$ cuando son soportados sobre $\mathrm{TiO}_{2}$ no se observa en el caso de los óxidos de Mn. Este hecho puede ser debido a la presencia de electrones d en los óxidos de Mn, hecho que no ocurre en los óxidos de V, Mo y W. Por tanto, la interacción del $\mathrm{TiO}_{2}$ está altamente relacionada con la presencia o ausencia de electrones d en el catión metálico de la fase soportada.

\section{AGRADECIMIENTOS}

Los autores agradecen la financiación de este trabajo a la OTAN (CRG-960316) y a la Junta de Castilla y León (Sa37/98). JMGA agradece, en especial, al MEC la concesión de una beca, FPI, postdoctoral.

\section{BIBLIOGRAFÍA}

1. P.E. Marti, M. Maciejewski and A. Baiker, Appl. Catal., B: Environ. 4, 225 (1994).

2. G. Groppi, M. Bellotto, C. Cristiani and P. Forzatti, Appl. Catal., A: General 104, 101 (1993).

3. H.G. Lintz and K. Wittstock, Catal. Today 29, 457 (1996).

4. U.S. Ozkan, R.F. Kueller and E. Moctezuma, Ind. Eng. Chem. Res. 29, 1136 (1990).

5. J. Carnö, M. Ferrandon, E. Björnbom and S. Järäs, Appl. Catal., A: General 155, 265 (1997).

6. G.K. Boreskov, B.I. Popov, V.N. Bibin and E.S. Kozishnikova, Kinet. Katal. 9, 796 (1968).

7. J.E. Germain and R. Perez, Bull. Soc. Chim. France, 4683(1972).

8. C, Lahousse, A. Bernier, A. Gaigneaux, P. Ruiz, P. Grange and B. Delmon, in 3rd world congress on Oxidation Catalysis, R.K. Grasselli et al. eds., Elsevier, Amsterdam, 1997, p. 777.

9. J.E. Germain and R. Perez, Bull. Soc. Chim. France, 541 and 2042 (1972).

10.M. Baldi, V. Sanchez-Escribano, J.M. Gallardo-Amores, F. Milella and G. Busca, App. Catal., B, Environ. 17, L175 (1998)

11. M. Baldi, E. Finocchio, F. Milella, and G. Busca, Appl. Catal.,B: Environ. 16, 43 (1998).

12. A. Barresi, I. Mazzarino and B. Ruggeri, Chim. Ind. (Milano) 71(3), 64 (1989); M.F.M. Zwinkels, S.G. Jaras, P.G. Menon and T.A. Griffin, Catal. Rev. Sci. Eng. 35, 319 (1993)

13. A. Wollner, F. Lange, H. Schmelz and H. Knozinger, Appl. Catal., A: General 94, 181 (1993)

14. W.S. Kiljlstra, E.K. Poels, A.Bliek, B.M. Weckhuysen and R.A. Schoonheydt, J. Phys. Chem. B 101, 309 (1997).

15. "Vanadia catalysts for selective oxidation of hydrocarbons and their derivatives", B. Grzybowska-Swierkosz, F. Trifirò and J.C. Vedrine, eds., App. Catal. A: General 157, 1-426 (1997).

16 J.M. Gallardo, V.S. Escribano and G. Busca, J. Mater. Chem. 5(8), 1245 (1995). 17 H.D. Lutz, B. Muller and H.J. Steiner, J. Solid State Chem. 90, 54 (1991).

18 G. Busca, G. Ramis, J.M. Gallardo-Amores, V. Sánchez Escribano and P. Piaggio, J. Chem. Soc., Faraday Transactions 90, 3181 (1994).

Recibido: $21-10-98$

Aceptado: 13-2-99 